Materials and Methods To complete this work we used human cancer and non-malignant cell lines as well as primary human malignant liquid (leukaemia) and solid tumour samples. We also used human primary natural killer (NK) cells and TALL104 cytotoxic $\mathrm{T}$ cell line. Western blot analysis, on-cell and in-cell Western analysis, ELISA, quantitative real-time PCR, flow cytometry (including imaging flow cytometry) and biochemical enzyme activity were used as research tools.

Results We found that galectin-9 is highly expressed in human liquid (acute myeloid leukaemia (AML) and solid (breast, colorectal, brain etc.) tumour cells. G protein-coupled receptors of latrophilin family and their natural ligand fibronectin leucine rich transmembrane protein 3 (FLRT3) trigger externalisation/exocytosis, and, in some cases (e. g. AML), biosynthesis of galectin-9 and its receptor and possible trafficker Tim-3. Galectin- 9 can be used to suppress anti-cancer immune responses by impairing cytotoxic activity of NK cells and killing $\mathrm{T}$ cells.

Conclusions We report the Tim-3-galectin-9 secretory pathway as one of the biochemical mechanisms operated by human cancer cells to escape host immune surveillance. Differential activities based on cell type of origin are discussed.

Disclosure Information I.M. Yasinska: None. E. Klenova: None. E. Klenova Fasler-Kan: None. V. Sumbayev: None.

\section{P01.21 VALIDATION OF ROMO1 INHIBITORS AS THE MITOCHONDRIAL ROS ENHANCER FOR ANTICANCER DRUG DEVELOPMENT}

${ }^{1} Y$ Yoo*, ${ }^{1} \mathrm{D}$ You, ${ }^{2} \mathrm{~J}$ Park, ${ }^{3} \mathrm{H}$ Kim. ${ }^{1}$ Korea University College of Medicine, Seoul, Korea, Republic of; ${ }^{2}$ Korea Institute of Radiological and Medical Sciences, Seoul, Korea, Republic of; ${ }^{3}$ Yonsei University College of Medicine, Seoul, Korea, Republic of

\subsection{6/jitc-2020-ITOC7.33}

Background Chemotherapy in conjunction with surgical operations have been commonly used for the treatment of many tumors. However, a significant number of tumors fail respond to radiation therapy and/or chemotherapy because many forms of tumors appear to become less sensitive or resistant to radiation and anticancer drugs after consecutive treatments. Although extensive studies on the molecular mechanisms of resistance to chemo- and/or radiation therapy have been carried out, problems related to overcoming this resistance remain to be solved. Romo1 is a nuclear-encoded small transmembrane protein located in mitochondrial inner membrane. It is known to induce mitochondrial reactive oxygen species (ROS) production in response to various cellular stresses. For a decade, Romo1 has been studied in the context of mitochondrial ROS production, cancer cell invasion, inflammation, replicative senescence, and mitochondrial dynamics.

Materials and Methods We identified a Romo1 antagonist and tried to its efficacy as chemotherapy sensitizer using cancer cells and animal models.

Results A Romo1 antagonist can enhance the cellular levels of ROS, leading to tumor cell death. Its treatment induced the elevation of chemotherapy-induced oxidative damage of cancer cells. We also treated the Romo1 antagonist in combination with various chemotherapeutic agents.

Conclusions We suggest that Romo1 antagonist can enhance the cellular levels of ROS, leading to elevation of chemotherapy-induced oxidative damage of cancer cells. We also suggest that Romo1 is the new target to identify effective substances for development of chemotherapy sensitizer.
Disclosure Information Y. Yoo: None. D. You: None. J. Park: None. H. Kim: None.

\section{P01.22 EXTENDING CAR T CELL THERAPY APPLICATIONS VIA DRUG INDUCIBLE CONTROL OF TRANSGENE EXPRESSION}

${ }^{1} \mathrm{~B}$ Kotter*, ${ }^{1} \mathrm{~N}$ Werchau, ${ }^{2} \mathrm{~W}$ Krueger, ${ }^{2} \mathrm{~A}$ Roy, ${ }^{1} \mathrm{~J}$ Mittelstaet, ${ }^{1} \mathrm{~A}$ Kaiser. ${ }^{1}$ Miltenyi Biotec B.V. and Co. KG, Bergisch Gladbach, Germany; ${ }^{2}$ Lentigen Technology Inc., Gaithersburg, MD, USA

\subsection{6/jitc-2020-ITOC7.34}

Background Adoptive transfer of chimeric antigen receptor (CAR)-modified T cells has emerged as a promising treatment modality for a broad range of cancers highlighted by the approval of Kymriah $^{\mathrm{TM}}$ and Yescarta ${ }^{\mathrm{TM}}$ for the treatment of $\mathrm{B}$ cell malignancies. However, lack of control of CAR $T$ cell function and consequent excessive inflammation in patients can result in severe side effects especially when targeting tumor-associated rather than tumor-specific antigens. Thus, temporal and tunable control of CAR activity is of major importance for the clinical translation of innovative CAR designs. While the activation of suicide switches results in the apoptotic elimination of the transferred cells, other strategies, e.g. anti-tag CARs or small molecule-gated CARs, enable the reversible control of CAR-mediated function at the protein level but are restricted to a particular CAR design. Focusing on the control of expression rather than CAR signaling, transcriptional regulators represent a versatile tool facilitating a wide range of CAR T cell applications.

Materials and Methods To maintain control over the infused CAR $T$ cell product and mitigate risks for the patient, we describe here the development of an inducible switch system for the transcriptional regulation of transgene expression in primary, human $\mathrm{T}$ cells. Chemically regulated synthetic transcription factors composed of a zinc finger DNA-binding domain, an inducible control domain and a transcription activation domain were designed, screened for functionality, and evaluated in $\mathrm{T}$ cells regarding their potential to control CAR expression both in vitro and in vivo.

Results By screening, we identified a synthetic transcription factor, which shows high transcriptional output in $\mathrm{T}$ cells in the presence of a clinically relevant inducer drug and absence of background activity in the non-induced state. Using this system we were able to control the expression of a CAR recognizing the CD20 antigen present on B cells and B cell leukemic blasts. The addition of the inducer drug resulted in rapid expression of the anti-CD20 CAR on the $\mathrm{T}$ cell surface. Moreover, inducible anti-CD20 CAR T cells executed cytolytic activity against CD20 positive target cells and secreted cytokines upon stimulation in vitro. Effectivity in co-cultures was thereby comparable to $\mathrm{T}$ cells expressing the anti-CD20 CAR under a conventional constitutive promoter. Furthermore, we could fine-tune CAR activity by titrating the inducer concentration. By defining the time-point of induction, modulation of the onset of therapy was achieved. Upon inducer drug discontinuation, inducible CD20 CAR T cells lost CAR expression and concurrently all CAR-related functions, indicating that the 'on' and 'off' status can be tightly controlled by the administration of the drug. After pausing of CAR T cell-mediated activity, we could re-induce CAR expression suggesting complete reversibility of effector function. Finally, we were able to show that inducible CD20 CAR T cells mediate a 
significant, strictly inducer-dependent antitumor activity in a well-established mouse model of B cell lymphoma.

Conclusions The zinc-finger-based transcriptional control system investigated in this study provides small molecule-inducible control over a therapeutically relevant anti-CD20 CAR in primary $\mathrm{T}$ cells in a time- and dose-dependent manner. The tight regulation of CAR expression will pave the way for safer cellular therapies.

Disclosure Information B. Kotter: A. Employment (full or parttime); Significant; Miltenyi Biotec B.V. \& Co. KG. N. Werchau: A. Employment (full or part-time); Significant; Miltenyi Biotec B.V. \& Co. KG. W. Krueger: A. Employment (full or part-time); Significant; Lentigen Technology Inc. A. Roy: A. Employment (full or part-time); Significant; Lentigen Technology Inc. J. Mittelstaet: A. Employment (full or part-time); Significant; Miltenyi Biotec B.V. \& Co. KG. A. Kaiser: A. Employment (full or part-time); Significant; Miltenyi Biotec B. V. \& Co. KG.

\section{P01.23 CREATING A CELL-CULTURE BASED REPORTER SYSTEM FOR THE EVALUATION OF MOLECULAR SIGNALING MECHANISMS OF INHIBITORY CHIMERIC ANTIGEN RECEPTORS}

MA Funk*, A De Sousa Linhares, C Battin, K Radakovics, J Leitner, P Steinberger. Medical University of Vienna, Vienna, Austria

\subsection{6/jitc-2020-ITOC7.35}

Background Adoptive transfer of $\mathrm{T}$ cells expressing chimeric antigen receptors (CARs) is a novel treatment option for patients with B-cell lineage derived cancers. Other cancer entities are less successfully targeted due to the lack of antigens that are expressed on cancer cells but not healthy tissue cells. One way to address this issue is the concept of inhibitory chimeric antigen receptors (iCARs) that deliver an inhibitory signal upon antigen encounter on off-target cells. The activating effect of CARs depends on a multitude of factors including expression levels, affinity, different signaling domains and steric effects. It has to be expected that co-expression of an iCAR would result in even more complexity. Consequently, there is a demand for a robust high-throughput cellular system to evaluate iCAR-formats.

Materials and Methods Our approach is based on a previously published Jurkat based triple parameter reporter cell (TPR) system. This setup allows for molecular monitoring of the $\mathrm{T}$ cell activation state by measuring fluorescent reporter gene expression via flow cytometry. Inhibitory effects of receptors are determined as the ratio of cellular geometric mean fluorescent intensity (gMFI) in the presence of the inhibitory ligand versus without inhibitory ligand.

Results To test if inhibitory receptors would measurably reduce reporter activation, PD-1 as a well-characterized inhibitory receptor was expressed on Jurkat TPRs. When PD-L1 was present during stimulation reporter activation was reduced by 26-34\% proving feasibility of our approach. Intracellular domains of other inhibitory receptors including BTLA, ILT-2 and KIR2DL1 were evaluated. All three domains outperformed PD-1 in a series of experiments with a mean reduction of gMFI by $48-57 \%, 50-53 \%$ and $38-41 \%$ respectively. To assess if the reporter platform could be used to study downstream signaling pathways of inhibitory constructs we created a SHP-2 knock-out reporter cell line using the
CRISPR/Cas9 gene editing technique. To simulate different degrees of activating signal strength, peptide-MHC complex recognizing activating receptors were created. The reporter activation correlated with the concentrations of peptide in the stimulation cultures. Co-expression of iCAR and CAR could be achieved using selection for two separate antibiotic resistance genes introduced into the respective vector. Preliminary experiments showed greatly reduced inhibitory efficacy of iCAR molecules due to an adhesion effect resulting from the high affinity extracellular domain of the iCARs that lead to tighter cell-cell contact and stronger stimulation through the CAR.

Conclusions We present a highly flexible and controllable Jurkat-based reporter cell platform for the thorough study of inhibitory signaling mechanisms. This project was supported by the Austrian Science Fund, FWF; Project P32411

Disclosure Information M.A. Funk: None. A. De Sousa Linhares: None. C. Battin: None. K. Radakovics: None. J. Leitner: None. P. Steinberger: None.

\section{P01.24 THE SELECTIVE HDAC6 INHIBITOR ITF3756 INCREASES THE DIFFERENTIATION TO CENTRAL MEMORY T CELLS WITH REDUCED EXHAUSTION PHENOTYPE}

C Ripamonti, C Steinkuhler, G Fossati*. Italfarmaco SpA, Cinisello Balsamo, Italy

\subsection{6/jitc-2020-ITOC7.36}

Background Central memory $\mathrm{T}$ cells show superior persistence and antitumor immunity compared to effector memory and effector $\mathrm{T}$ cells. $\mathrm{T}$ effector cells respond quickly to tumors, but they are terminally differentiated and undergo apoptosis upon killing activity. $\mathrm{T}$ memory differentiate rapidly into $\mathrm{T}$ effector cells and maintain a pool of cells that can continuously differentiate thus sustaining a more lasting response. In adoptive cell therapy (ACT), T cells infused into patients may have a limited time of activity if they are terminally differentiated, and may rapidly undergo exhaustion and apoptosis. The development of new strategies based on novel agents able to generate memory $\mathrm{T}$ cells ex-vivo is important for a successful clinical application of ACT.We have studied the effect of a potent and selective HDAC6 inhibitor, ITF3756, on CD8 T cells differentiation during an in vitro induced exhaustion process.

Materials and Methods To induce exhaustion purified human CD8 + cells were stimulated twice with anti-CD3/CD28 beads (1:2) during 5 days, with or without ITF3756 $1 \mu \mathrm{M}$ or $2 \mu \mathrm{M}$ added at all times of stimulation. At day 3 and 5 the expression of exhaustion, memory and effector $\mathrm{T}$ cells markers were analyzed by flow cytometry. Cells were also collected at day 5 for genes expression analysis. Expression of exhaustion, $\mathrm{T}$ phenotype, metabolic pathway and inflammatory cytokines were investigated by qPCR. Paired two-tailed t-tests was used to determine statistical significance between control versus treatment group at day 3 and 5 in 10 different donors. P-values $\leq 0.05$ were considered significant.

Results ITF3756 $1 \mu \mathrm{M}$ increased significantly the $\mathrm{T}$ central memory phenotype (CD45RO+CD62L +CCR7+) and decreased significantly the $\mathrm{T}$ effector phenotype (CD45RO +CD62L-CCR7-). The expression of CD62L in $\mathrm{T}$ central memory cells was significantly increased in agreement with the high expression of this marker in naïve and memory $\mathrm{T}$ cells. ITF3756 treatment decreased significantly the expression of 